\title{
Symmetries, holography, and quantum phase transition in two-dimensional dilaton AdS gravity
}

\author{
Mariano Cadoni, ${ }^{*}$ Matteo Ciulu, ${ }^{\dagger}$ and Matteo Tuveri ${ }^{\ddagger}$ \\ Dipartimento di Fisica, Università di Cagliari and INFN, \\ Sezione di Cagliari, Cittadella Universitaria, 09042 Monserrato, Italy
}

(Received 18 December 2017; published 29 May 2018)

\begin{abstract}
We revisit the Almheiri-Polchinski dilaton gravity model from a two-dimensional (2D) bulk perspective. We describe a peculiar feature of the model, namely the pattern of conformal symmetry breaking using bulk Killing vectors, a covariant definition of mass and the flow between different vacua of the theory. We show that the effect of the symmetry breaking is both the generation of an infrared scale (a mass gap) and to make local the Goldstone modes associated with the asymptotic symmetries of the 2D spacetime. In this way a nonvanishing central charge is generated in the dual conformal theory, which accounts for the microscopic entropy of the $2 \mathrm{D}$ black hole. The use of covariant mass allows to compare energetically the two different vacua of the theory and to show that at zero temperature the vacuum with a constant dilaton is energetically preferred. We also translate in the bulk language several features of the dual CFT discussed by Maldacena et al. The uplifting of the $2 \mathrm{D}$ model to $(d+2)$-dimensional theories exhibiting hyperscaling violation is briefly discussed.
\end{abstract}

DOI: 10.1103/PhysRevD.97.103527

\section{INTRODUCTION}

Two-dimensional (2D) dilaton gravity models have a long history (see Ref. [1] for a review). They have been first proposed for studying quantum gravity in a simplified context [2]. Later, they have been developed along several different directions as an arena to understand gravity in a simplified setting, as effective description of the radial modes (the S-wave sector) of black holes [3] and as toy models for black hole evaporation and related information puzzles [4]. 2D dilaton gravity models have been also used to explore the anti-de Sitter/conformal field theory (AdS/ CFT) correspondence in two dimensions [5-8] and to investigate the microscopic origin of the BekensteinHawking black hole entropy [8].

Recently, a 2D AdS dilaton gravity model has been proposed by Almheiri and Polchinski (AP) [9]. The main motivation behind this proposal was to understand the infrared (IR) behavior of higher-dimensional black holes, which flow in the IR to an $\mathrm{AdS}_{2} \times S^{d}$ spacetime. Among many others, this is for instance the case of charged

\footnotetext{
*mariano.cadoni@ca.infn.it

†matteo.ciulu@gmail.com

matteo.tuveri@ca.infn.it
}

Published by the American Physical Society under the terms of the Creative Commons Attribution 4.0 International license. Further distribution of this work must maintain attribution to the author(s) and the published article's title, journal citation, and DOI. Funded by SCOAP.
Reissner-Nordström black holes in general relativity. The IR behavior of these black holes is problematic for several reasons. In fact, the $T=0$ extremal black hole is a zero temperature vacuum state with non vanishing entropy and the backreaction is so strong that there are no finite energy excitations above the vacuum [10-12].

The AP dilaton gravity model coupled to a matter field $f$ is described by the action [9] (we set the dimensionless Newton constant to $G_{2}=1 / 8 \pi$ )

$S=\int d^{2} x\left[\frac{1}{2} \sqrt{-g}(\eta R-V(\eta))+\mathcal{L}_{m}\right]+\int d t \mathcal{L}_{b}$,

where $\eta$ is a scalar field (the dilaton). The matter and boundary Lagrangian $\mathcal{L}_{m}, \mathcal{L}_{b}$ are given by

$$
\begin{aligned}
\mathcal{L}_{m} & =-\frac{1}{8} \sqrt{-g}(\nabla f)^{2}, \\
\mathcal{L}_{b} & =\sqrt{|h|} \eta \mathcal{K},
\end{aligned}
$$

where $h_{i j}$ is the induced metric on the boundary and $\mathcal{K}$ is the trace of the second fundamental form. The potential for the dilaton is

$$
V(\eta)=2 \lambda^{2}\left(\alpha^{2}-\eta\right)
$$

Notice that the potential contains a dimensionless parameter $\alpha^{2}$ and a parameter $\lambda$ with dimensions $[L]^{-1}$. 
The AP model extends the well-known Jackiw-Teitelboim (JT) model [2], characterized by a simple homogeneous potential, by including the constant term $2 \lambda^{2} \alpha^{2}$.

The most important feature of the AP model is that it allows for two kinds of vacuum AdS solutions. One with constant dilaton, pure $\mathrm{AdS}_{2}$, to which we will refer as constant dilaton vacuum (CDV). The other one is a solution with a nonconstant, linearly varying, dilaton which we will call linear dilaton vacuum (LDV). When uplifted to $(d+2)$-dimensions, these vacuum solutions produce different spacetimes. The CDV produces a spacetime of the form $\mathrm{AdS}_{2} \times R^{d}$, i.e., an intrinsically $2 \mathrm{D}$ spacetime. On the other hand, the uplifting of the LDV leads to a hyperscaling violating geometry $H^{d+2}$ [13], which describes the warping of $\mathrm{AdS}_{2}$ with $R^{d}$. In this case, the dilaton plays the role of the radius of $R^{d}$.

The JT model has been widely used as a 2D toy model for higher-dimensional black holes, to give a microscopic interpretation of black entropy [8] and to understand the AdS/CFT correspondence in 2D in terms of asymptotic symmetries $[5,6,14]$. Conversely, the extended AP model has been recently used as a description of extremal black holes in 2D (in particular to investigate the breakdown of semiclassical thermodynamics and the flow from LDV to CDV). Moreover it has also been used to describe the backreaction on holographic correlators $[9,10]$ and to investigate its relation with the conformal symmetry breaking [15].

In this paper we present a revisitation of the AP model from a $2 \mathrm{D}$ bulk perspective. The goal of our reconsinderation is twofold. On the one hand, we want to describe the pattern of conformal symmetry breaking and to explain its dynamical consequences (generation of an IR scale and the appearance of Goldstone modes) focusing mainly on bulk gravitational features of the solutions. On the other hand, we would like to connect and translate the formulation of the boundary theory in terms of the Schwarzian action of Ref. [15] in the language of Refs. [5,6,14], i.e., in the language of canonical realization of the asymptotic symmetry group of $\mathrm{AdS}_{2}$.

We will show that the pattern of conformal symmetry breaking and its dynamical consequences can be simply described using bulk Killing vectors, the covariant (bulk) mass definition of Refs. [16-18] and the flow between a "symmetry-respecting" vacuum and a "symmetry-violating" vacuum. In this way we can easily understand, from a purely 2D bulk gravitational perspective, the generation of an IR scale (the mass gap/scale of conformal symmetry breaking in the conformal correlators) and the appearance of local Goldstone modes.

Our bulk perspective will also allows us to compare, energetically, the CDV and LDV vacuum. We will show that whereas at nonvanishing temperature the LDV is always energetically preferred, at $T=0$ the situation is reversed and the CDV is favorite. This signals a $T=0$ quantum phase transition which, from an higher-dimensional perspective, can be thought as a spontaneous dimensional reduction from a $d+2$ to $d=2$ dimensions spacetime, whose possible role in quantum gravity has been emphasized in Ref. [19].

The structure of the paper is the following. In Sec. II we revisit the various solutions of the AP model. In Sec. III we define the covariant mass and explain its leading role as the physical mass of the solutions. In Sec. IV we discuss the symmetries of the model, the pattern of the conformal symmetry breaking as well as their consequences for the boundary theory. In Sec. V we discuss the free energy of the solutions and the quantum phase transition. In Sec. VI we present our conclusions. In the Appendix we discuss the uplifting of a $2 \mathrm{D}$ model to a $(d+2)$-dimensions model exhibiting hyperscaling violation in the ultraviolet (UV).

\section{SOLUTIONS AND VACUA}

In Schwarzschild coordinates and in absence of matter $(f=0)$, owing to $2 \mathrm{D}$ Birkhoff theorem, the most general solution of the model (2) is a two-parameter family of solutions,

$$
\begin{aligned}
d s^{2} & =-\left(\lambda^{2} x^{2}-a^{2}\right) d t^{2}+\left(\lambda^{2} x^{2}-a^{2}\right)^{-1} d x^{2}, \\
\eta & =\alpha^{2}+\eta_{0} \lambda x .
\end{aligned}
$$

where $a^{2}$ and $\eta_{0}$ are dimensionless integration constants. Being our solution asymptotically AdS, we can use the standard ADM procedure to assign a mass to it [20]. The mass defined in this way, i.e., the ADM mass, has the physical meaning of the energy of the gravitational configuration measured with respect to the reference $\mathrm{AdS}$ background solution [20].

The ADM mass of the solution depends on both $\eta_{0}$ and $a$ and on the parameter $\lambda$ [9]

$$
M_{\mathrm{ADM}}=\frac{\eta_{0} \lambda a^{2}}{2} .
$$

It is important to stress that the ADM mass does not depend on the parameter $\alpha^{2}$ appearing in the AP potential (3) for the dilaton.

An important feature of the AP model is that it allows for two different vacuum solutions, i.e., solutions with $M_{\mathrm{ADM}}=0$. In fact, for $\eta_{0}=0$ and $\alpha^{2} \neq 0$ we have the constant dilaton vacuum. It describes the $\mathrm{AdS}_{2}$ spacetime with a constant dilaton. It is already well known that at classical level this vacuum does not allow for finite energy excitations [9-11]. This is immediately evident from Eq. (5): for $\eta_{0}=0, M_{\mathrm{ADM}}$ identically vanishes, independently from the value of $a$. Conversely, for $\eta_{0} \neq 0$ we have the linear dilaton vacuum, which is $\mathrm{AdS}_{2}$ endowed with a linear dilaton. Differently from the CDV, this vacuum allows for continuous excitations with $a^{2}>0$.

It is important to stress that we have two different LDV depending on the value of the parameters $\left(\eta_{0}, a\right)$ and $\alpha$. 
For $\alpha^{2}=0$ the AP model reduces to the JT model and the solution for the dilaton is linear and homogeneous. Notice that this is not an exact solution of the AP model (i.e., the model with $\alpha^{2} \neq 0$ ) but appears only as an asymptotic solution for $x \rightarrow \infty$. On the other hand, for $\eta_{0} \neq 0$ and $\alpha^{2} \neq 0$, the LDV is an exact solution of the AP model and interpolates between the CDV at small $x$ and a linear, homogeneous dilaton at large $x$. Whenever the distinction between these two LDV will be necessary, we will call the LDV with $\alpha^{2} \neq 0$ interpolating linear dilaton vacuum (ILDV).

If one uses $M_{\mathrm{ADM}}$ as the mass of the solution the three vacua become completely degenerate. They all have $M_{\mathrm{ADM}}=0$, as it is evident from Eq. (5), because the CDV is characterized by $\eta_{0}=0$, whereas the LDV and the ILDV have $a=0$. Actually, in view of the physical meaning of the ADM mass, the three vacua are degenerate by definition. This means that they represent three different sectors of the theory, which strictly speaking cannot be compared.

The finite, $M_{\mathrm{ADM}}>0$, excitations of the LDV and ILDV can be interpreted as $2 \mathrm{D}$ black holes with horizon radius $x=a / \lambda$ and temperature and entropy given by

$$
\begin{gathered}
T=\frac{\lambda a}{2 \pi}, \quad S=2 \pi \eta_{h}=2 \pi \alpha^{2}+2 \pi \eta_{0} a, \\
M_{\mathrm{ADM}}=\frac{2 \pi^{2} \eta_{0}}{\lambda} T^{2} .
\end{gathered}
$$

Notice that the interpretation of the $a^{2}>0$ solutions as $2 \mathrm{D}$ black holes is not completely straightforward. In fact, it is well known that the metric (4) can be brought by a coordinate transformation in the maximally extended form $d s^{2}=-\cosh ^{2} \rho d \tau^{2}+\frac{d \rho^{2}}{\lambda^{2}},-\infty<\tau, \rho<+\infty$, which describes full, geodesically complete $\mathrm{AdS}_{2}$ (see e.g., Ref. [21]). This is not anymore true if one takes into account the fact that points where the dilaton vanishes have to be considered spacetime singularities. This makes solutions with different $a^{2}$ as globally inequivalent and allows for the interpretation of the $a^{2}>0$ solution as a $2 \mathrm{D}$ black hole [22].

The previous argument forbids the existence of 2D black hole solutions with constant dilaton, in agreement with the absence of finite energy excitations of the CDV. On the other hand, we can formally consider zero mass thermal excitation of the CDV of the form

$$
\begin{aligned}
d s^{2} & =-\left(\lambda^{2} x^{2}-\frac{4 \pi^{2} T^{2}}{\lambda^{2}}\right) d t^{2}+\left(\lambda^{2} x^{2}-\frac{4 \pi^{2} T^{2}}{\lambda^{2}}\right)^{-1} d x^{2}, \\
\eta & =\alpha^{2} .
\end{aligned}
$$

This solution can be obtained from the CDV by a coordinate transformation, which generates a horizon with related temperature $T$. Because in the $\mathrm{CDV}$ there is no spacetime singularity, there is no obstruction to extend (8) beyond the horizon, to cover the whole CDV spacetime. Thus, the solution (8) is geometrically equivalent to the CDV, but can be formally used to describe zero mass thermal excitation of the CDV.

The discussion of the spacetime singularities is much simpler using light-cone coordinates $x^{ \pm}$. Using the $S L(2, R)$ isometric transformations, the solution (4) becomes

$$
\begin{aligned}
d s^{2} & =-\frac{4}{\lambda^{2}\left(x^{+}-x^{-}\right)^{2}} d x^{+} d x^{-}, \\
\eta & =\alpha^{2}+\frac{2 \frac{\eta_{0}}{\lambda}-M_{\mathrm{ADM}} x^{+} x^{-}}{x^{+}-x^{-}} .
\end{aligned}
$$

The $\eta=0$ singularity is located at

$$
\left(x^{+}+\frac{\alpha^{2}}{M_{\mathrm{ADM}}}\right)\left(x^{-}-\frac{\alpha^{2}}{M_{\mathrm{ADM}}}\right)=\frac{\frac{2 \eta_{0}}{\lambda} M_{\mathrm{ADM}}-\alpha^{4}}{M_{\mathrm{ADM}}^{2}},
$$

whereas the timelike asymptotic boundary of $\mathrm{AdS}_{2}$ is located at $x^{+}=x^{-}=t= \pm \sqrt{\frac{2 \eta_{0}}{\lambda M_{\mathrm{ADM}}}}$. The nature of the singularity depends on the value of $M_{\mathrm{ADM}}$. For $M_{\mathrm{ADM}}>\lambda \alpha^{4} /\left(2 \eta_{0}\right)$ the singularity is spacelike whereas for $M_{\mathrm{ADM}}<\lambda \alpha^{4} /\left(2 \eta_{0}\right)$ it is timelike.

For planar spatial topology the ILDV gives a nice, effective, 2D description of the flow from an $\mathrm{AdS}_{2} \times R^{d^{1}}$ geometry in the IR to a hyperscaling violating geometry [13] in $(d+2)$-dimension in the $\mathrm{UV}$, of which $\operatorname{AdS}_{d+2}$ is a particular case. From the 2D perspective this flow is a simple consequence of both the relation $R \propto \eta^{p}$ between the dilaton and the radius $R$ of $R^{d}$ and of the constant/linear behavior of the dilaton at small/large $x$. We briefly discuss the uplifting of the AP model to a $(d+2)$-dimensional theory with hyperscaling violation in the Appendix.

From the thermodynamical point of view, the CDV gives the typical $T=0$, extremal, state with non vanishing entropy of a large class of $(d+2)$-dimensional extremal black holes, like e.g., charged Reissner-Nordström black holes in four dimensions. Near extremality, the mass-temperature relation for the excitations, $M_{\mathrm{ADM}} \propto T^{2}$ in Eq. (7), implies the breakdown of the thermodynamical semi-classical description and the appearance of a mass gap $[10,12]$

$$
M_{\text {gap }}=\frac{\lambda}{2 \pi^{2} \eta_{0}} .
$$

The generation of the mass gap is the quantum counterpart of the absence of finite energy excitations of the CDV [11], which in turns is related to the strong backreaction on $\mathrm{AdS}_{2}$. From the AdS/CFT correspondence point of view, the appearance of the mass gap (11) can be also explained in terms of the pattern of breakdown of the conformal symmetry which generates, in the IR, a mass scale of order $\lambda[9,10,15]$.

\footnotetext{
${ }^{1} \mathrm{AdS}_{2} \times S^{d}$ in the case of spherical spacial topology.
} 
Despite the successes of the AP model described above, two aspects are still not completely clear. The first is the characterization of the energy of the solution trough the ADM mass (5). This mass does not distinguish between the different vacua of the theory, in fact it is independent from $\alpha$. Due to the different features of the two vacua, the ADM-mass degeneracy between the CDV and LDV becomes particularly ambiguous. Moreover, $M_{\mathrm{ADM}}$ does not keep informations about the presence of the mass gap. Last but not least, it also does not seem a suitable physical parameter to characterize the singularity. Indeed, the transition between spacelike and timelike singularities occurs not when $M_{\mathrm{ADM}}$ changes sign, as expected, but rather at strictly positive values.

The second aspect is the characterization of the pattern of conformal symmetry breaking. This pattern has been described using correlation functions in the dual conformal field theory. However, in the spirit of the AdS/CFT correspondence one should be able to characterize completely this pattern also using only the 2D gravity theory. In what follows, we will show how the peculiarities of dilaton gravity in 2D spacetime can help us to clarify the picture presented above.

\section{COVARIANT MASS}

The first peculiarity of $2 \mathrm{D}$ dilaton gravity is that the metric always admits the existence of a Killing vector whose explicit form depends on the dilaton $[14,16,18]$

$$
\chi^{\mu}=F_{0} \epsilon^{\mu \nu} \partial_{\nu} \eta,
$$

where $F_{0}$ is a normalization factor. The second is the existence of a covariant, conserved mass [16,17]

$$
M=-\frac{F_{0}}{2}\left[\int{ }^{\eta} V(s) d s+(\nabla \eta)^{2}\right] .
$$

In this paper we will use the normalization prescription of Refs. [16,18], i.e., $F_{0}=\left(\lambda \eta_{0}\right)^{-1}$.

The covariant mass $M$ gives a definition of the energy of the solution, which is invariant under Weyl transformation of the metric [18]. It may differ from the standard ADM mass only by a constant (temperature independent) term. For this reason it is particularly appropriate to quantify the energy of the different vacua of the AP model.

Computing the covariant mass for the general solution (4) of our model we get,

$$
M=\frac{a^{2} \eta_{0} \lambda}{2}-\frac{\alpha^{4} \lambda}{2 \eta_{0}}=M_{\mathrm{ADM}}-\frac{\alpha^{4} \lambda}{2 \eta_{0}} .
$$

There are several reasons indicating that the covariant mass $M$ and not the ADM mass $M_{\mathrm{ADM}}$ has to be considered as the physical mass of the solutions. For $\alpha=0$ we have $M=M_{\mathrm{ADM}}$. By using $M$ instead of $M_{\mathrm{ADM}}$ we remove the degeneracy between the CDV and the LDV and keep also track about the nonexistence of finite energy excitations of the CDV. The ILDV has negative energy $M=-\frac{\alpha^{4} \lambda}{2 \eta_{0}}$, whereas for the CDV we have $M \rightarrow-\infty$. Moreover, the right-hand side (r.h.s.) of Eq. (10) can be written as $2 \eta_{0} M / M_{\mathrm{ADM}}^{2}$. Thus, the spacetime singularity is spacelike for $M>0$, whereas it becomes timelike for $M<0$.

\section{SYMMETRIES AND SYMMETRY BREAKING}

Let us now discuss the symmetries of the different vacua of the AP model. The isometry group of $\mathrm{AdS}_{2}$ is the $S L(2, R) \sim S O(1,2)$ group generated by three Killing vectors. In Schwarzschild coordinates (4) they represent time translations $\mathcal{T}$, dilatations and special conformal transformations. However, the $S L(2, R)$ symmetry is only a symmetry of the metric. The whole solution contains also the dilaton which, under isometric transformations generated by the Killing vector $\chi$, transforms as $\delta \eta=\mathcal{L}_{\chi} \eta=$ $\chi^{\mu} \partial_{\mu} \eta$ [14]. Notice that a constant dilaton will preserve the $S L(2, R)$ symmetries of the metric, whereas a non constant dilaton will necessarily break explicitly the $S L(2, R)$ symmetry. On the other hand, the $2 \mathrm{D}$ metric allows for the killing vector (12), which is also always a symmetry of the dilaton $(\delta \eta=0)$. Thus, a nonconstant dilaton breaks explicitly the full $S L(2, R)$ symmetry group of $\mathrm{AdS}_{2}$ down to its subgroup $H$ generated by the Killing vector (12).

In the case of the static solutions (4) the residual symmetry is the time translations $\mathcal{T}$ and the symmetry breaking pattern is $S L(2, R) \rightarrow \mathcal{T}$ [14]. As a consequence, the CDV of the AP model preserves the full $S L(2, R)$ symmetries of $\mathrm{AdS}_{2}$, whereas the LDV breaks $S L(2, R) \rightarrow \mathcal{T}$. In this way we can describe the IR/UV flow CDV $\rightarrow$ LDV as a symmetry breaking of the full $S L(2, R)$ group down to time translations.

The parameter controlling the symmetry breaking is $\partial_{x} \eta=\eta_{0} \lambda$. Any $\eta_{0} \neq 0$ value breaks the $S L(2, R)$ symmetry to $\mathcal{T}$ and generates a mass-scale in the IR, set by $\lambda$, which is of the same order of magnitude of the mass gap (11).

Thus, the presence of a nonconstant dilaton breaks the conformal symmetry of the $\mathrm{AdS}_{2}$ background and generates in the quantum regime a mass gap through $\eta_{0} \neq 0$. Further, it also affects the asymptotic symmetries of $\mathrm{AdS}_{2}$ [14] and the dynamics of the boundary theory. In particular, the latter can be constructed using boundary curves $t(u)$, where $u$ is the time coordinate in the one-dimensional regularized boundary of $\mathrm{AdS}_{2}$ [15].

Actually, the two descriptions, that of Refs. [5,14], which uses canonical realization of the asymptotic symmetry group (ASG) of $\mathrm{AdS}_{2}$ and that of Ref. [15] give similar results but using different languages and a different coordinate system.

The ASG of $\mathrm{AdS}_{2}[6,14]$ is given by reparametrizations of the type $\xi^{t}=\epsilon(t), \xi^{x}=x \epsilon^{\prime}(t)$ and is generated by a 
single copy of the Virasoro algebra. This transformations map the boundary curves $t(u)$ into curves $t(u)=u+\epsilon(u)$. On the other hand, they act on the asymptotic expansion of the metric $[5,8,14]$

$$
\begin{aligned}
& g_{t t}=-\lambda^{2} x^{2}+\gamma_{t t}(t)+o\left(x^{-2}\right), \\
& g_{t x}=\frac{\gamma_{t x}(t)}{\lambda^{3} x^{3}}+o\left(x^{-5}\right), \\
& g_{x x}=\frac{1}{\lambda^{2} x^{2}}+\frac{\gamma_{x x}(t)}{\lambda^{4} x^{4}}+o\left(x^{-6}\right),
\end{aligned}
$$

by transforming the values of the boundary fields $\gamma$.

In the case of the CDV (which is called "pure $\mathrm{AdS}_{2}$ " in Ref. [15]), the dilaton is constant, $\eta_{0}=0$, and we do not have the explicit breaking of the $S L(2, R)$ conformal symmetry. The full Virasoro ASG is spontaneously broken by the $\mathrm{AdS}_{2}$ bulk geometry down to the $S L(2, R)$ group of isometries. The zero modes can be characterized either by the boundary curves $t(u)$ (in the language of Ref. [15]) or by the boundary deformations $\gamma$ (in the language of Ref. [6,14]). These zero modes can be viewed as the Goldstone modes associated to the spontaneous breaking of the ASG [15]. However, these modes are not local, there is no local action one can write for them and this is related to the fact that the central charge $c$ in the Virasoro algebra is zero.

In the case of the LDV (which is called "nearly $\mathrm{AdS}_{2}$ " in Ref. [15]), as discussed above, the non constant value of the dilaton breaks explicitly $S L(2 R) \rightarrow \mathcal{T}$. This gives, in the language of Ref. [15] a new dimensional coupling constant, the renormalized boundary value of the dilaton $\phi_{r}(u)$, which can be used to constrain the shape of $t(u)$ and to produce a local, Schwarzian action for the pseudoGoldstone bosons $t(u)$.

Conversely, in the language of Refs. [6,14], the explicit breaking of the conformal symmetry is described by the asymptotic expansion for the dilaton $\eta=\eta_{0} \rho(t) x+$ $o\left(x^{2}\right)$ with the boundary field $\rho(t)$ transforming as $\delta \rho=$ $\epsilon \dot{\rho}+\dot{\epsilon} \rho$ under the action of the ASG $[6,14]$.

The two boundary fields can be identified: $\phi_{r}(u)=\eta_{0} \rho(t(u))$. In both descriptions the physical effect of the explicit symmetry breaking is to make the Goldstone modes local and to generate a nonvanishing central charge in the Virasoro algebra,

$$
c=12 \eta_{0},
$$

through the anomalous transformation of the boundary stress energy tensor $T_{t t}$ under the action of the ASG. In fact, we have $T_{t t}^{(1)}=\phi_{r}\{t(u), u\}$ for the boundary theory of Ref. [15], whereas $T_{t t}^{(2)}=-2 \eta_{0} / \lambda \ddot{\rho}$ for the boundary theory of Refs. $[6,14]$. The central charge $c$ takes the form given by Eq. (18) if we choose $\rho=1$. We can always make this choice by fixing the $u$-reparametrization in the boundary which corresponds, in the language of Refs. [6,14], to consider deformations of the dilaton near the on-shell solution (see $[6,14]$ for details).

The stress energy tensor $T_{t t}^{(2)}$ can be brought in the form $T_{t t}^{(1)}$. In fact, by considering finite transformations associated with the infinitesimal ones characterized by $\epsilon=u$, by using the transformation of the boundary field $\rho$ and by setting $\rho=1$ one finds $T_{t t}^{(2)}=(c / 12)\{t(u), u\}$. The link between the origin of Schwarzian action and the presence of a non constant dilaton was emphasized also in Ref. [23], where it was shown that in a holographic framework the effective action of the AP model can be put in a Schwarzian form using the anomalous trace Ward identity. In particular, the anomaly turns out to be proportional to the source of the scalar operator dual to the dilaton, which is the analogue of our function $\rho(t)$.

Summarizing, the explicit breaking of the conformal symmetry, $S L(2, R) \rightarrow \mathcal{T}$ generated by a nonconstant dilaton has two effects. First, it generates at the quantum level an IR scale in the form of mass gap, $M_{\text {gap }} \sim \lambda$, separating the CDV from the LDV.

Second, it transforms the global Goldstone modes of the CDV associated with the spontaneous breaking of the ASG into local pseudo-Goldstone modes, producing a central charge $c=12 \eta_{0}$ in the Virasoro algebra associated to the ASG. This central charge therefore counts the number of pseudo-Goldstone modes. From this perspective we can identify the degrees of freedom responsible for the entropy of the 2D dilatonic black hole as these pseudo-Goldstone modes. The microscopic derivation of the entropy of the 2D dilatonic black hole given in Ref. [5] can be seen as counting the states of these modes. It is also interesting to notice that the mass gap in the chiral 2D CFT can be also understood as finite size effect generated by a plane/ cylinder transformation of the vacuum of a CFT with nonvanishing central charge $c=12 \eta_{0}[24,25]$.

\section{QUANTUM PHASE TRANSITION AND SPONTANEOUS DIMENSIONAL REDUCTION}

The AP model allows for two different class of solutions, namely the 2D black hole (4) and the zero mass thermal excitations of the CDV (8). One important question is to determine which of these two solutions is, from the thermodynamic point of view, globally favorite.

Using the 2D Hamiltonian formalism [7], this can be done by computing the difference $\Delta F$ between the free energy, $F$, of the two solutions. In the case under consideration this computation is not straightforward because $\Delta F$ is usually computed for solutions having the same asymptotical behavior.

The presence of the dilaton makes the asymptotics of the two classes of solutions of the AP model (linear and constant dilaton, respectively) different, thus preventing the standard computation of $\Delta F$. This problem can be 
circumvented by defining the free energy of the solution with respect to its own vacuum [26]. This method has been applied, for example, in Ref. [26] to calculate $\Delta F$ for two classes of 4D solutions approaching asymptotically to AdS and to a solution with hyperscaling violation, respectively.

Using this prescription for the free energy $F$ in the Euclidean action formalism, for the case under consideration we get:

$$
\begin{aligned}
F^{\mathrm{BH}} & =-\frac{2 \pi^{2} \eta_{0}}{\lambda} T^{2}-2 \pi \alpha^{2} T, \\
F^{T} & =-\kappa \eta_{h}^{C}=-2 \pi \alpha^{2} T, \\
\Delta F & =F^{\mathrm{BH}}-F^{T}=-\frac{2 \pi^{2} \eta_{0}}{\lambda} T^{2},
\end{aligned}
$$

where $F^{\mathrm{BH}}$ is the free energy of the 2D black hole (4) obtained by subtracting the contribution of the ILDV, whereas $F^{T}$ is the free energy of the thermal excitation of the CDV obtained by subtracting the contribution of its own vacuum. From Eq. (19) follows immediately that for any $T \neq 0, \Delta F<0$ and the $2 \mathrm{D}$ dilatonic black hole is energetically preferred.

By construction, Eq. (19) does not give any information about the behavior at $T=0$, being $F$ defined with reference to the respective vacua at $T=0$. Formally, at $T=0$ the two vacua are degenerate, consistently with the degeneracy of the CDV and ILDV when the ADM mass is used to characterize the two solutions.

Moreover, the semi-classical approximation, on which the euclidean action formalism is based, breaks down at $T \sim M_{\text {gap }}$, so that Eq. (19) cannot be trusted at $T=0$.

At $T=0$ there is no thermal contribution to the free energy and $\Delta F$ is given by the mass difference between the two vacua, $\Delta F_{T=0}=\Delta M=M^{\mathrm{ILDV}}-M^{\mathrm{CDV}}$.

We have already argued that we should use the covariant mass (14) as the physical mass instead of the ADM mass. Using this mass in the computation we find $\Delta F_{T=0} \rightarrow$ $\infty>0$. This means that the CDV is energetically preferred and that at $T=0$ the 2D dilatonic black hole undergoes a quantum phase transition to the CDV. Let us note that, here, we are referring to the usual thermodynamical meaning of phase transitions. In fact, we are considering a thermodynamical system, which can exist in two different configurations, the black hole given by Eq. (4) and the thermal excitations of the CDV given by (8). Comparing the free energy of the two configurations at the same temperature we discover that at $T=0$ the free energy of the black hole is bigger than that of the CDV. This means that at zero temperature the black hole undergoes a phase transition to the CDV. We call this phase transition "quantum" because it happens at zero temperature and can be fully understood only at full quantum level. The free energy of the CDV diverges. In fact, in the limit $\eta \rightarrow 0$ the covariant mass (14) blows up and, classically, we can describe the phase transition as an instability of the ILDV in which the CDV expands to take over the spacetime. This description changes at quantum level, where the divergence of the covariant mass is cured by the presence of the mass gap. However, there is no reason to expect in this quantum description a change of sign of $\Delta F_{T=0}$.

From a four-dimensional perspective this quantum phase transition can be interpreted as a spontaneous dimensional reduction. In fact, the $(d+2)$-dimensional uplifting of the ILDV is a scale covariant geometry $H^{d+2}$ with hyperscaling violation in $(d+2)$-dimensions, whereas the uplifting of the $\mathrm{CDV}$ is $\mathrm{AdS}_{2} \times R^{d}$, i.e., a geometry which is intrinsically two-dimensional, being the radius of $R^{d}$ not dynamical. In terms of the uplifted geometries we have the $T=0$ phase transition $H^{d+2} \rightarrow \mathrm{AdS}_{2} \times R^{d}$.

This phase transition supports the suggestion of Ref. [19] about the existence of a spontaneous dimensional reduction of the spacetime to two dimensions near the Planck scale.

Let us conclude with some remarks about one loop corrections to the free energy (19). Our calculation is based on the semiclassical approximation. One loop corrections to $F$ have been shown in Refs. $[9,15]$ to have the typical $\log T$ behavior, which gives a dangerous divergent term in the IR. However, this term does not contribute to the entropy of the CDV [15], we therefore expect our result to extend also beyond the semi-classical approximation.

\section{CONCLUSIONS}

In this paper we have revisited the AP dilaton gravity model focusing mainly on bulk features of the model. Using a covariant definition of the mass, bulk Killing vectors and the flow between the two different vacua of the theory characterized, respectively, by a constant and linear varying dilaton, we have given a description of the pattern of conformal symmetry breaking, which is complementary to that emerging in the dual CFT [15].

This pattern is quite similar to that pertinent to hyperscaling-violating geometries in higher dimensions, to which we show the AP model can be uplifted. In fact, as a result of the flow between a "symmetry-violating" vacuum and a "symmetry-respecting" vacuum at the quantum level an IR scale is generated in the form of a mass gap. The other effect of the conformal symmetry breaking is to make local the Goldstone modes associated with the asymptotic symmetries of the 2D spacetime. This generates a nonvanishing central charge in the dual conformal theory, which explains at microscopic level the entropy of the 2D black hole [5].

We have also shown that several features of the boundary theory described in Ref. [15] can be easily translated in our language, which is based on bulk quantities and on the asymptotic symmetries of the spacetime.

Finally, the use of the covariant mass as measure of the energy of the solutions, allowed us to compare energetically 
the two different vacua of the theory, showing the existence of a zero temperature phase transition in which the vacuum with constant dilaton is energetically preferred. We speculate that this quantum phase transition could be related to the spontaneous dimensional reduction of the spacetime to two dimensions near the Planck scale described in Ref. [19].

\section{APPENDIX UPLIFTING TO $d+2$ DIMENSIONS}

Let us briefly show how the solution (4) can be uplifted to a $(d+2)$-dimensional geometry describing the flow from a $\mathrm{AdS}_{2} \times R^{d}$ geometry in the IR to a $(d+2)$-dimensional geometry with hyperscaling violation in the UV.

In $(d+2)$-dimensions the model is described by the action

$$
S=\int d^{d+2} x \sqrt{-g_{(d+2)}} R_{(d+2)}+\mathcal{L}_{M},
$$

where $L_{M}$ is the Lagrangian for matter fields, which may also contains explicit coupling of matter fields to the dilaton.

For simplicity we assume that after dimensional reduction to two spacetime dimensions, the term $\mathcal{L}_{M}$ either reproduce exactly the potential (3) or a potential, which can be approximated by (3). We look for brane solutions of the model, i.e., solutions for which the $d$-dimensional spatial sections have planar topology $R^{d}$ and the dilaton, $\eta$, plays the role of the radius: $d s_{(d+2)}^{2}=d s_{(2)}^{2}+\eta^{2 / d} d x_{i} d x^{i}$. In general, the dimensional reduction of the action (A1) on this background produces kinetic terms for the dilaton $\eta$ in the $2 \mathrm{D}$ dilaton gravity action. This terms can be put to zero by a Weyl rescaling of the 2D metric [18]. This corresponds to use, instead, the dimensional reduction

$$
d s_{(d+2)}^{2}=\eta^{\frac{1-d}{d}} d s_{(2)}^{2}+\eta^{\frac{2}{d}} d x_{i} d x^{i}
$$

One can check that the dimensional reduction now produces the AP action (1). Using Eq. (A2) and the form of the $2 \mathrm{D}$ solution given by Eq. (4), one can easily realize that the $(d+2)$-dimensional solution interpolates between an hyperscaling violating geometry at large $x$ and an $\mathrm{AdS}_{2} \times R^{d}$ geometry at small $x$. In fact, for $x \rightarrow \infty$ the term proportional to $x$ in the dilaton dominates, and the change of radial coordinate, $x \propto r^{-2 d /(d+1)}$, brings the metric in the scale covariant form given in Ref. [13]

$$
d s_{(d+2)}^{2}=r^{-2 \frac{d-\theta}{d}}\left(-r^{-2(z-1)} d t^{2}+d r^{2}+d x_{i} d x^{i}\right)
$$

The hyperscaling violating parameter $\theta$ and the dynamical exponent $z$ are

$$
\theta=\frac{d(d-1)}{d+1}, \quad z=\frac{2 d}{d+1}
$$

Conversely, in the near horizon limit the term proportional to $x$ in the dilaton, can be neglected with respect to the constant term and the metric (A2) gives an $\mathrm{AdS}_{2} \times R^{d}$ geometry. This can be also considered as the limiting case $\theta=0, z=\infty$ of the hyperscaling violating geometry (A3).
[1] D. Grumiller, W. Kummer, and D. V. Vassilevich, Dilaton gravity in two-dimensions, Phys. Rep. 369, 327 (2002).

[2] R. Jackiw, Lower dimensional gravity, Nucl. Phys. B252, 343 (1985).

[3] A. Strominger, Les Houches lectures on black holes, arXiv: hep-th/9501071.

[4] C. G. Callan, Jr., S. B. Giddings, J. A. Harvey, and A. Strominger, Evanescent black holes, Phys. Rev. D 45, R1005 (1992).

[5] M. Cadoni and S. Mignemi, Asymptotic symmetries of AdS (2) and conformal group in $d=1$, Nucl. Phys. B557, 165 (1999).

[6] M. Cadoni, P. Carta, D. Klemm, and S. Mignemi, AdS(2) gravity as conformally invariant mechanical system, Phys. Rev. D 63, 125021 (2001).

[7] M. Caldarelli, G. Catelani, and L. Vanzo, Action, Hamiltonian and CFT for 2-D black holes, J. High Energy Phys. 10 (2000) 005.

[8] M. Cadoni and S. Mignemi, Entropy of 2-D black holes from counting microstates, Phys. Rev. D 59, 081501 (1999).
[9] A. Almheiri and J. Polchinski, Models of $\mathrm{AdS}_{2}$ backreaction and holography, J. High Energy Phys. 11 (2015) 014.

[10] A. Almheiri and B. Kang, Conformal symmetry breaking and thermodynamics of near-extremal black holes, J. High Energy Phys. 10 (2016) 052.

[11] J. M. Maldacena, J. Michelson, and A. Strominger, Antide Sitter fragmentation, J. High Energy Phys. 02 (1999) 011.

[12] J. Preskill, P. Schwarz, A. D. Shapere, S. Trivedi, and F. Wilczek, Limitations on the statistical description of black holes, Mod. Phys. Lett. A 06, 2353 (1991).

[13] X. Dong, S. Harrison, S. Kachru, G. Torroba, and H. Wang, Aspects of holography for theories with hyperscaling violation, J. High Energy Phys. 06 (2012) 041.

[14] M. Cadoni and S. Mignemi, Symmetry breaking, central charges and the AdS(2) / CFT(1) correspondence, Phys. Lett. B 490, 131 (2000).

[15] J. Maldacena, D. Stanford, and Z. Yang, Conformal symmetry and its breaking in two dimensional Nearly Anti-de-Sitter space, Prog. Theor. Exp. Phys. 2016, 12C104 (2016). 
[16] R. B. Mann, Conservation laws and 2-D black holes in dilaton gravity, Phys. Rev. D 47, 4438 (1993).

[17] J. Gegenberg, G. Kunstatter, and D. Louis-Martinez, Classical and quantum mechanics of black holes in generic 2-d dilaton gravity, arXiv:gr-qc/9501017.

[18] M. Cadoni, Conformal equivalence of 2-D dilaton gravity models, Phys. Lett. B 395, 10 (1997).

[19] S. Carlip, Spontaneous dimensional reduction?, AIP Conf. Proc. 1483, 63 (2012).

[20] L. F. Abbott and S. Deser, Stability of gravity with a cosmological constant, Nucl. Phys. B195, 76 (1982).

[21] A. Achucarro and M. E. Ortiz, Relating black holes in twodimensions and three-dimensions, Phys. Rev. D 48, 3600 (1993).
[22] M. Cadoni and S. Mignemi, Nonsingular four-dimensional black holes and the Jackiw-Teitelboim theory, Phys. Rev. D 51, 4319 (1995).

[23] M. Cvetic and I. Papadimitriou, $\mathrm{AdS}_{2}$ holographic dictionary, J. High Energy Phys. 12 (2016) 008; Erratum 01 (2017) 120(E).

[24] M. Cadoni and M. Cavaglia, Open strings, 2-D gravity and AdS/CFT correspondence, Phys. Rev. D 63, 084024 (2001).

[25] M. Cadoni and P. Carta, 2-D black holes, conformal vacua and CFTs on the cylinder, Phys. Lett. B 522, 126 (2001).

[26] M. Cadoni and S. Mignemi, Phase transition and hyperscaling violation for scalar black branes, J. High Energy Phys. 06 (2012) 056. 